\title{
Asymmetric PML for the Absorption of Waves. Application to Mesh Refinement in Electromagnetic Particle-In-Cell Plasma Simulations. ${ }^{1}$
}

\author{
J.-L. Vay a J.-C. Adam ${ }^{\text {b A. Héron }}{ }^{\text {b }}$ \\ ${ }^{a}$ Lawrence Berkeley National Laboratory, CA, USA \\ ${ }^{\mathrm{b}}$ CPHT, Ecole Polytechnique, France
}

\begin{abstract}
We present an extension of the Bérenger Perfectly Matched Layer with additional terms and tunable coefficients which introduce some asymmetry in the absorption rate. We show that the discretized version of the new PML offers superior absorption rates than the discretized standard PML under a plane wave analysis. Taking advantage of the high rates of absorption of the new PML, we have devised a new strategy for introducing the technique of Mesh Refinement into electromagnetic Particle-In-Cell plasma simulations. We present the details of the algorithm as well as a 2-D example of its application to laser-plasma interaction in the context of fast ignition.
\end{abstract}

Key words: Mesh refinement; Particle-In-Cell; plasma; absorbing boundary condition

\section{Introduction}

We present an extension of the original Bérenger formulation of the Perfectly Matched Layer (1) with additional terms and tunable coefficients. Under certain constraints on the coefficients, the newly introduced "medium" does not generate any reflection at any angle or any frequency and is thus a Perfectly Matched Layer (PML). Unlike the original PML, however, the new formulation introduces some asymmetry in the absorption rate and is therefore labeled APML for Asymmetric Perfectly Matched Layer.

$\overline{1 \text { Work }}$ performed for USDOE under Contracts DE-AC03-76F00098 at UC-LBNL.

Preprint submitted to Elsevier Science 24 October 2003 
Taking advantage of the high rates of absorption of the APML, we have devised a new strategy for introducing the technique of Mesh Refinement into electromagnetic Particle-In-Cell plasma simulations. Previous attempts have relied on algorithms of various complexity to connect calculations of EM fields at the border of regular grids at different resolutions (we will refer these as "sewing" algorithms). Most were unstable at small wavelengths while the stable ones suffered form inherent limitations on their ability to avoid spurious wave reflection at interfaces and were complicated to implement (6). Instead, we propose a technique by substitution. We present the details of the algorithm as well as a 2-D example of its application to laser-plasma interaction in the context of fast ignition.

\section{Definition of the APML}

This section summarized results presented in (5), in which the reader can find a complete presentation of the APML.

For the transverse electric (TE) case, we define the APML as

$$
\begin{aligned}
\varepsilon_{0} \frac{\partial E_{x}}{\partial t}+\sigma_{y} E_{x} & =\frac{c_{y}}{c} \frac{\partial H_{z}}{\partial y}+\bar{\sigma}_{y} H_{z} \\
\varepsilon_{0} \frac{\partial E_{y}}{\partial t}+\sigma_{x} E_{y} & =-\frac{c_{x}}{c} \frac{\partial H_{z}}{\partial x}+\bar{\sigma}_{x} H_{z} \\
\mu_{0} \frac{\partial H_{z x}}{\partial t}+\sigma_{x}^{*} H_{z x} & =-\frac{c_{x}^{*}}{c} \frac{\partial E_{y}}{\partial x}+\bar{\sigma}_{x}^{*} E_{y} \\
\mu_{0} \frac{\partial H_{z y}}{\partial t}+\sigma_{y}^{*} H_{z y} & =\frac{c_{y}^{*}}{c} \frac{\partial E_{x}}{\partial y}+\bar{\sigma}_{y}^{*} E_{x} \\
H_{z} & =H_{z x}+H_{z y}
\end{aligned}
$$

For $c_{x}=c_{y}=c_{x}^{*}=c_{y}^{*}=c$ and $\bar{\sigma}_{x}=\bar{\sigma}_{y}=\bar{\sigma}_{x}^{*}=\bar{\sigma}_{y}^{*}=0$, this system reduces to the Bérenger PML medium, while adding the additional constraint $\sigma_{x}=\sigma_{y}=\sigma_{x}^{*}=\sigma_{y}^{*}=0$ leads to the system of Maxwell equations in vacuum.

It can be shown (5) that if $c_{x}=c_{x}^{*}, c_{y}=c_{y}^{*}, \bar{\sigma}_{x}=\bar{\sigma}_{x}^{*}, \bar{\sigma}_{y}=\bar{\sigma}_{y}^{*}, \frac{\sigma_{x}}{\varepsilon_{0}}=\frac{\sigma_{x}^{*}}{\mu_{0}}$ and $\frac{\sigma_{y}}{\varepsilon_{0}}=\frac{\sigma_{y}^{*}}{\mu_{0}}$ then the impedance of an APML medium is $Z= \pm \sqrt{\mu_{0} / \varepsilon_{0}}$, which is the impedance of vacuum. Hence, like the PML, given some restrictions on the parameters, the APML does not generate any reflection at any angle and any frequency. Just as for the PML, this property is not retained after discretization (5).

We assume that we have an APML layer of thickness $\delta$ (measured along $x$ ) 
and that $\sigma_{y}=\bar{\sigma}_{y}=0$ and $c_{y}=c$. The coefficient of reflection given by this layer is

$$
R_{A P M L}(\theta)=e^{-2\left(\sigma_{x} \cos \varphi / \varepsilon_{0} c_{x}\right) \delta}
$$

which happens to be the same as the PML theoretical coefficient of reflection if we assume $c_{x}=c$. Hence, it follows that for the purpose of wave absorption, the term $\bar{\sigma}_{x}$ seems to be of no interest. However, although this conclusion is true at the infinitesimal limit, it does not hold for the discretized counterpart. Using plane wave analysis, we show that our APML implementations lead to higher absorption rates than the considered PML implementations (Fig.1).

\section{Mesh refinement by substitution}

We propose a technique by substitution using the following procedure (see Fig. 2):

(1) a usual EM-PIC calculation is performed on a grid G at resolution R1,

(2) in an area A where one is interested in a higher resolution R2, we perform an electromagnetic PIC calculations on

(a) a patch $\mathrm{P} 1$ at resolution $\mathrm{R} 1$,

(b) a patch $\mathrm{P} 2$ at resolution $\mathrm{R} 2$,

both covering $\mathrm{A}$ and being terminated by an APML ABC,

(3) the EM force F acting on a macroparticle is $F(G)$ outside $A$ and is $F(G)$ $\mathrm{F}(\mathrm{P} 1)+\mathrm{F}(\mathrm{P} 2)$ inside, where $\mathrm{F}(\alpha)$ means the force resulting from the fields computed on grid (or patch) $\alpha$.

The immediate advantage of the procedure over a more standard grid "sewing" technique is its simplicity since no special (and often cumbersome) algorithm is needed at grid interfaces. Also, since it allows the use of very efficient absorbing boundary conditions, it offers very high order wave absorption at any angle at the interface, while other algorithms are usually limited to first or second order absorption algorithms with respect to wavelength and angle.

One possible drawback of this technique is the fact that inside a refined area, the field calculation is performed three times: one in the fine resolution patch, one in the coarse resolution patch and one on the main grid in the refined area. However, for a refinement factor of two, the additional cost in the patch (compared to a sewing method) is $50 \%$ in 2-D and $25 \%$ in 3-D. An additional amount of work also comes from the use of absorbing layers. We considered both of these costs acceptable, given the potential benefits and the simplicity offered by the proposed method. 


\subsection{Current deposition and force gathering}

Another potential source of additional work comes from the current deposition and force gathering onto and from the grids which may have to be done on the three grids in the refined area. However, if the gather and scatter use linear weightings (the standard in PIC calculations), the current depostion needs to be done on the fine grid only and then propagated to the coarse patch and main grid; likewise, the field can be summed from every grid to an auxilliary grid of resolution R2 and then gathered onto particles. In this way, there is no penalty on the expensive gather/scatter part and only minimal additional work.

\subsection{Entrance and exit of macroparticles}

A question which arises with this method concerns the treatment of the macroparticles entering and exiting the patches P1 and P2. Ideally, the macroparticles should enter (exit) a patch and their corresponding field should magically appear (vanish) as if the particle was coming from (departing to) infinity. These conditions may be challenging to achieve and we have opted for an operationally simple procedure: the current of a macroparticle $\mathrm{M}$ of charge $\mathrm{q}$ is deposited inside a patch as soon as it enters it and stops being deposited as soon as it leaves the refined area. The consequence is the creation of a macroparticle of charge $-\mathrm{q}$ at the entrance point after the entrance of $\mathrm{M}$ and a macroparticle of charge $q$ standing at the exit point after the exit of $\mathrm{M}$. Although these are spurious charges that should not be present ideally, their effect should be minimal inside the patch because of the cancellation $F(P 2)$ $\mathrm{F}(\mathrm{P} 1)$.

Nevertheless, a residual spurious force is present due to this effect and it may be necessary to reduce it or, if possible, suppress it if it proves to be a problem. One method of suppressing the spurious force would consist of depositing the current inside the APML patch in a manner that prevents the appearance of these spurious charges. Another way would be to apply periodically a correction on the static part of the field using a "Boris" (2), "Marder-Langdon" (3), or hyperbolic (7) correction.

\section{Example of application to laser-plasma interaction}

We have implemented the new mesh refinement scheme in the 2-D electromagnetic Particle-In-Cell code Emi2d, developed at Ecole Polytechnique for study 
of laser-plasma interaction in the context of fast-ignition (4). In our test case (see Fig.3), a laser beam $\left(\lambda=1 \mu m, I=10^{20} W . \mathrm{cm}^{-2}, 2 \sigma=28 / k_{0}\right)$ impinges on a cylindrical plasma which density $\left(10 n_{c}\right)$ is far greater than the critical density $\left(n_{c}\right)$ and which initial temperature is $10 \mathrm{keV}$. The center of the plasma is artificially cooled to simulate a cold high-density core.

Two cases have been run. One case involves only one grid at high resolution and is the case of reference. The other one has one patch covering the all plasma target at the same resolution as the grid in the reference case, while the surrounding, including the region where the laser is launched into the simulation, is covered by a grid at lower resolution (a factor of two along each dimension). A selection of plots is displayed in Fig.4, which contains snapshots at (or near) the end of the simulations of the $X-Y$ and $X-V_{x}$ ions phasespace projections, as well as the field $B_{z}$, for the reference and the test (with refinement patch) cases. In the case with the refinement patch, the displayed $B_{z}$ contains the contributions of all grids in the patch area, as would be seen by a particle. We observe that the results are very similar in both cases, with or without the patch.

The biggest discrepancy is a residual of the laser which can be seen as if exiting the plasma on the $B_{z}$ plot in the test case, but actually exiting the refinement patch. This is obviously a non-physical effect since the laser should be totally absorbed by the plasma. The reason for this spurious residual of laser wave is as follows. The laser is launched outside the patch area and is thus not being propagated in the patch grids. When it hits the plasma, it triggers a response which generates a wave canceling out the incident wave. Due to different numerical relations of dispersion, the generated waves propagate at different velocities on each patch and do not cancel each other perfectly ${ }^{2}$. Whatever wave results from this imperfect cancellation acts on the plasma in the patched area and generates a response to it, which translates into a wave being launched on the main grid (verified on more detailed plots not displayed here). As expected physically, the sum of all these components is negligible in the plasma behind the laser impact front, as well as in the vacuum region behind the plasma which is enclosed in the patched area. Ouside the patch, only the components launched onto the main grid remain, giving the observed spurious residual of the laser wave. Since the area in which this spurious wave is present is of little effect on the rest of the simulation, this effect can be ignored in this case. In other applications where this effect may not be ignored, one may have to consider the use of Maxwell solvers which are less dispersive or devised an algorithm for a clean launch of the field residual at the patch boundary in the main grid, if possible.

$\overline{2}$ the cancellation of the incident laser wave can be seen as being cancelled out indenpendently by the plasma response on the main grid 


\section{Conclusion}

We have presented a new asymmetric PML (labeled APML) which is formally an extension of Bérenger's original formulation and have demonstrated that the discretized APML offers superior absorption rates than discretized PML under a plane wave analysis. Building upon this, we have devised a new strategy for implementing mesh refinement in electromagnetic Particle-In-Cell codes, relying on field substitution between patches terminated by an APML layer.

Preliminary results obtained from application to laser-plasma interaction in the context of fast ignition have shown very good agreements between a reference case without patch and a test case with one refinement patch. However, more studies are needed to fully explore the strentgh and limitations of this new method.

Although we have considered the finite-difference discretization of Maxwelllike equations only, the APML system of equations, as well as the new mesh refinement scheme, may be used with other discretization schemes, such as finite element, and may be applied to other wave equations for applications beyond electromagnetics.

\section{References}

[1] J.-P. Bérenger, "A Perfectly Matched Layer for the Absorption of electromagnetic waves", J. Comput. Phys. 114, 185 (1994)

[2] C. K. Birdsall and A. B. Langdon, Plasma Physics via Computer Simulation' (Adam Hilger, New York, 1991)

[3] A. B. Langdon, "On enforcing Gauss' law in electromagnetic particle-in-cell codes", Computer Phys. Comm. 70, 447 (1992)

[4] M. Tabak, J. Hammer, M. Eglinsky, W.L. Kruer, S.C. Wilks, J. Woodworth, E.M. Campbell, M.D. Perry, R.J. Mason "Ignition and high gain with ultrapowerful lasers", Phys. of Plasmas, vol. 1, issue 5, 1626 (1994)

[5] J.-L. Vay, "Asymmetric Perfectly Matched Layer for the Absorption of Waves", J. Comput. Phys. 183, 367 (2002)

[6] J.-L. Vay, "An extended FDTD scheme for the wave equation. Application to multiscale electromagnetic simulation", J. Comput. Phys. 167, 72 (2001)

[7] J.-L. Vay and C. Deutsch, "Charge compensated ion beam propagation in a reactor sized chamber", Phys. Plasma 51190 (1998) 


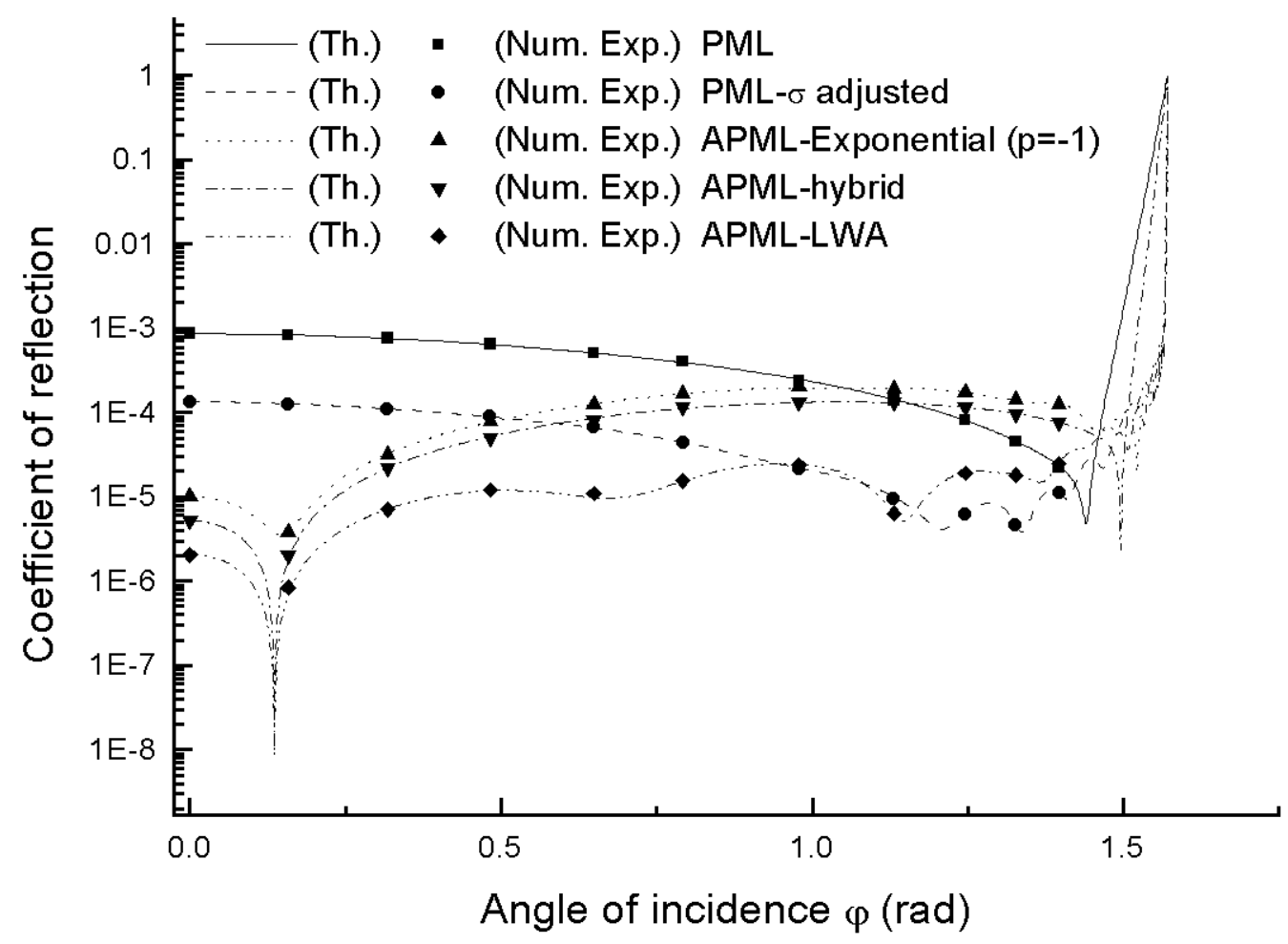

Fig. 1. Coefficient of reflection of various PML and APML implementations as a function of angle of incidence for a plane wave excited with a period $\tau=2 \pi / \omega=20 \delta x / c$ where $\delta \mathrm{x}$ is the mesh size. Theoretical (Th.) and numerical experiment (Num. Exp.) results are displayed. 


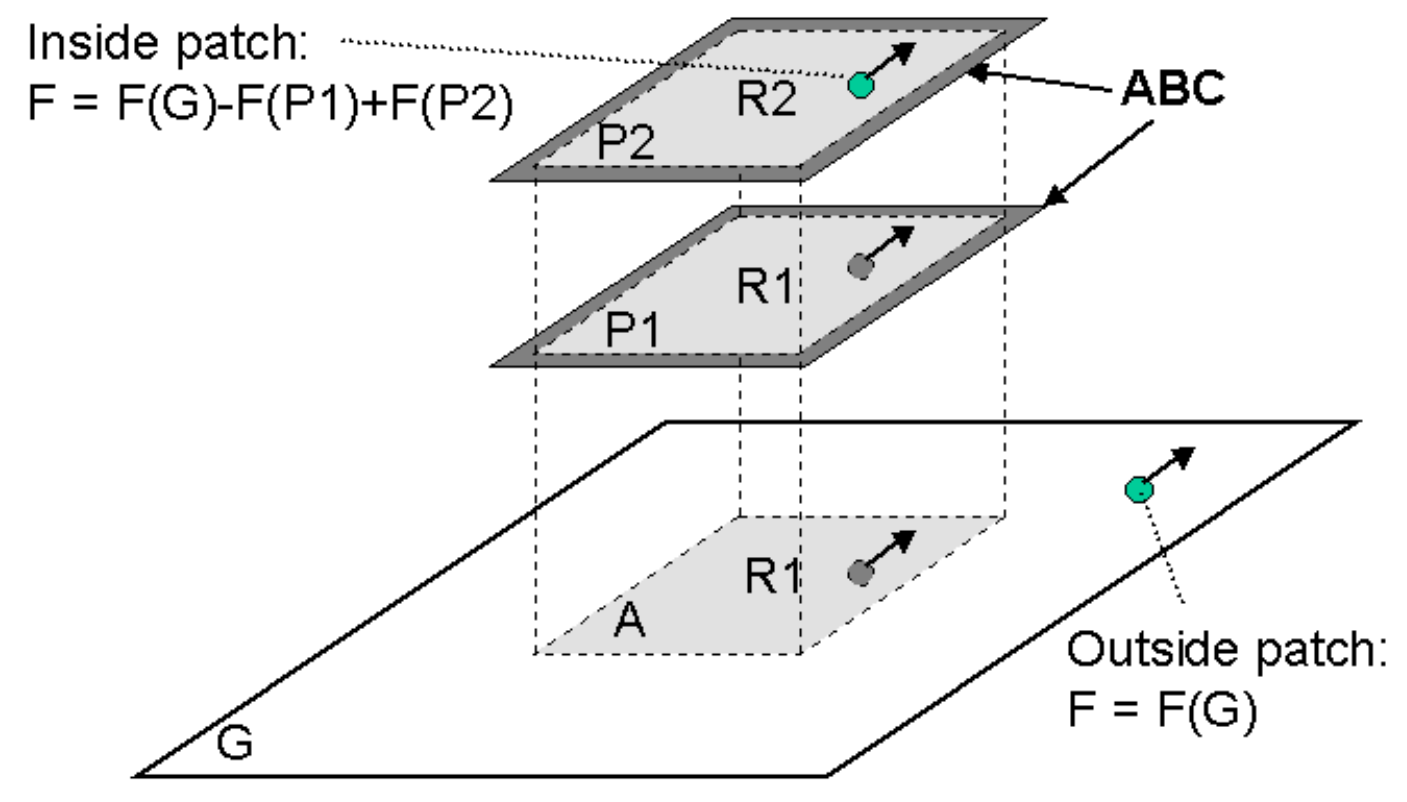

Fig. 2. Schematic of the grid structure for one refinement patch. It contains three grids: the main grid $\mathrm{G}$ (resolution R1), the grid P1 (resolution R1) and the grid P2 (resolution R2). The grid P1 and P2 are terminated by an APML absorbing boundary condition. 


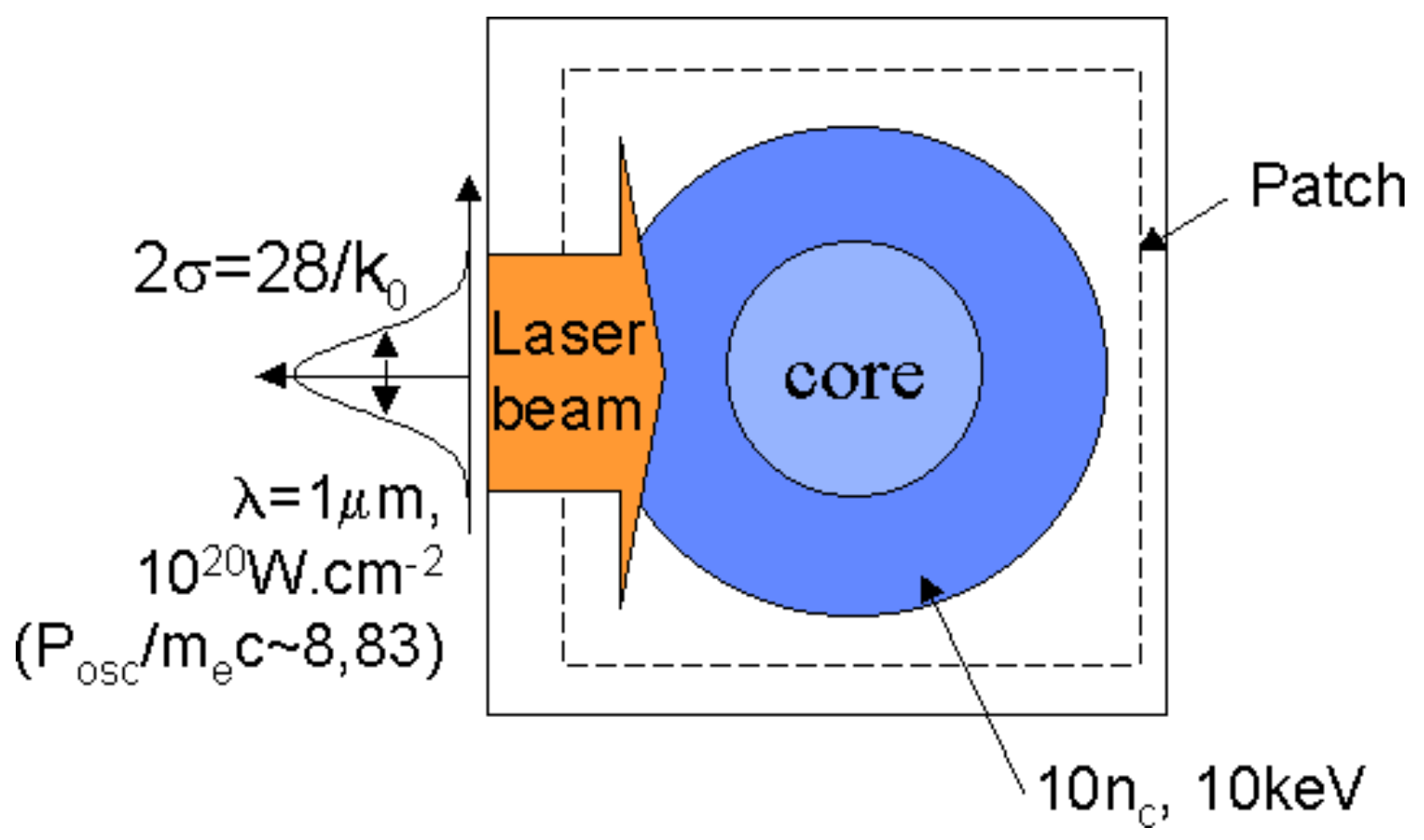

Fig. 3. Schematic of the test case. A laser impinges on a cylindrical target whose central part is artificially cooled to simulate a cold high-density core. The refinement patch encloses the entire target but the laser is launched outside the patch. 

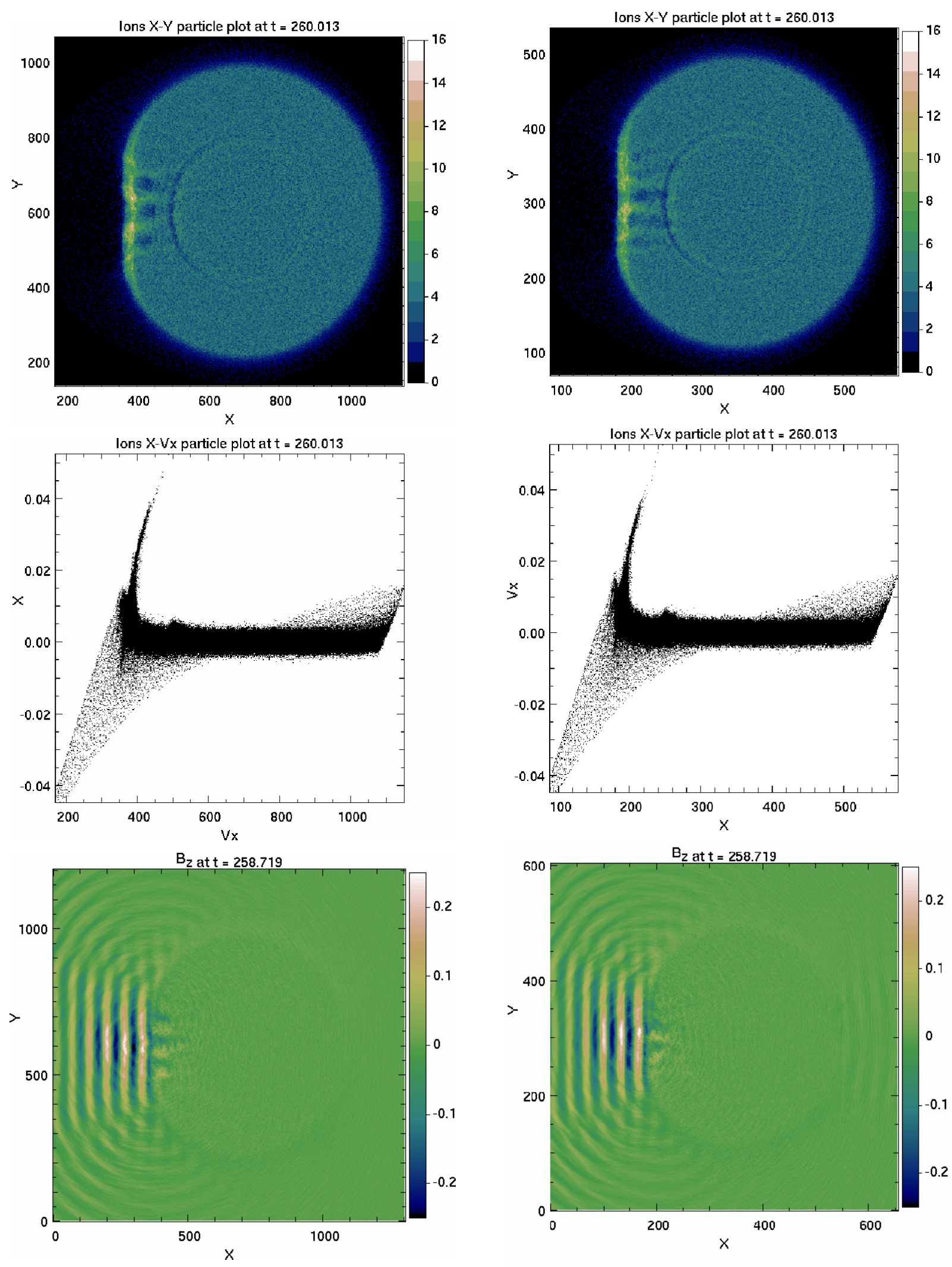

Fig. 4. $X-Y, X-V_{x}$ ions phase-space projections and $B_{z}$ for the case of reference (left column) and the case with one refinement patch (right column). 